\title{
Aplicação de Conceitos de Wayfinding em Interfaces Mobile de Recomendação de Rota
}

\author{
Maria Fernanda Castro, Patricia Tedesco
}
Centro de Informática - Universidade Federal de Pernambuco (UFPE)
50.733-970 - Recife - PE - Brasil
$\{m f c c, p c a r t\} @ c i n . u f p e . b r$

\begin{abstract}
Mobile technologies have allowed the development of several tools which assist users in their daily activities. Among them, route recommendation apps are gaining ground as powerful allies to urban mobility. However, with the growth of cities and complexity of their transport networks, providing mobility services has become a challenge, due to such amount of information in a relatively small smartphone screen. This paper proposes a mobile recommendation route prototype that uses Wayfinding techniques to represent mapping information, aiming at a more efficient and intuitive interface, as well as a validation experiment and its results.
\end{abstract}

Resumo. Tecnologias mobile permitiram o surgimento de diversas ferramentas que auxiliam usuários em tarefas diárias. Entre elas, aplicativos de recomendação de rota ganham espaço como poderosos aliados à mobilidade urbana. Porém, com o crescimento das cidades e complexidade de suas malhas de transporte, prover serviços de mobilidade tornou-se um desafio, devido à tamanha quantidade de informação na relativamente pequena tela de um smartphone. Este artigo propõe um protótipo de recomendação de rota mobile que utiliza técnicas da área de Wayfinding para representar dados do mapeamento, visando uma interface mais eficiente e intuitiva, bem como um experimento de validação e seus resultados.

\section{Introdução}

O surgimento das tecnologias mobile trouxe consigo uma nova visão sobre as interações humano-computador. Com o uso dos smartphones, e sua portabilidade, os sistemas tornaram-se capazes de acompanhar o usuário em suas atividades rotineiras, independente de sua localização. Assim, a relação humano-computador passou a se dar de forma atemporal, já que não havia mais a necessidade de um local específico para interagir com determinado aplicativo.

Vários sistemas mobile, então, concentraram-se num novo nicho de mercado: criar soluções que auxiliassem as pessoas, em tempo real, a executar pequenas tarefas cotidianas, como agendar encontros, pesquisar localizações ou até mesmo preços de restaurantes. Desta maneira, aplicativos de recomendação de rota (que já proliferavam em meio $W e b)$ tornaram-se poderosas ferramentas mobile, adquirindo novas funcionalidades e usos. Podemos citar, por exemplo, a capacidade de, ao utilizar a tecnologia GPS presente em dispositivos móveis, ajustar a rota sugerida simultaneamente ao deslocamento do usuário. 
Porém, apesar da tecnologia mobile trazer um novo horizonte para o desenvolvimento de aplicações, trouxe também alguns desafios. Um deles, como descrito em [1], é a limitação no tamanho e resolução de tela da maior parte dos smartphones disponíveis no mercado. A representação de uma enorme quantidade de informação em um espaço reduzido de tela torna-se um dos grandes problemas para a recomendação de rota, que faz uso, principalmente, de dados visuais complexos, como mapas e POIs (do inglês Points of Interest, Pontos de Interesse). Assim, corre-se o risco de tornar a interface de recomendação sobrecarregada, de difícil interpretação para 0 usuário.

Uma das saídas para contornar o problema pode residir na elaboração de sistemas de mapas mais simples, cujos elementos contribuam para despoluir a interface de recomendação. Neste contexto, podemos utilizar técnicas e conceitos presentes na área de Wayfinding, cujo foco reside na organização e representação do espaço e ambiente ao nosso redor [2].

Neste trabalho apresentaremos um protótipo de baixa fidelidade para aplicativos mobile de recomendação de rota que objetiva a construção de uma interface mais simples e intuitiva através da aplicação de princípios de Wayfinding nos elementos de mapa. A usabilidade deste protótipo foi avaliada por meio de experimentos realizados com um grupo de usuários, apresentando resultados satisfatórios e atestando a positiva inclusão das adaptações de Wayfinding na interface.

Este artigo está organizado no seguinte formato: A Seção 2 apresentará uma breve análise acerca da interface de alguns aplicativos móveis para recomendação de rota. A Seção 3 explorará princípios do Wayfinding que possam vir a ser aplicados em interfaces mobile. A seguir, será apresentada, na Seção 4, a metodologia utilizada na construção e validação do protótipo. A apresentação do protótipo e os testes de usabilidade encontram-se na Seção 5, seguida pelas conclusões e trabalhos futuros, Seção 6.

\section{Aplicativos de Recomendação de Rota}

Devido à demanda de um mercado cada vez maior, existem diversas opções de aplicativos mobile de recomendação de rota, com funcionalidades próprias e atuando sobre diferentes plataformas móveis.

Para o foco deste trabalho, foram escolhidas aplicações que apresentem diferentes interações entre usuário e seu sistema de mapas, a fim de uma melhor análise sobre a aplicação do Wayfinding em interfaces de recomendação. Uma interação entre usuário e mapa pode ser entendida como indo desde a representação de informação visual (por exemplo, o traçado da rota e a exibição do nome de vias) até funcionalidades dinâmicas (como a criação de novos Pontos de Interesse ao tocar a superfície do mapa).

\subsection{Google Maps}

Um dos mais populares aplicativos móveis de recomendação, o Google Maps [10] ajudou a disseminar este tipo de sistema entre os usuários de smartphone em todo o mundo. Além de possuir suporte tanto Web quanto mobile, uma API (Interface de 
Programação de Aplicativos) é disponibilizada para integrar as ferramentas de mapas com aplicações de terceiros.

As principais interações com o mapa, no Google Maps, incluem permitir a visualização do fluxo de tráfego nas vias (único para todos os tipos de veículo), visualizar a malha de alguns modais (ônibus, automóvel e bicicleta) e obter recomendações de rota para determinado modal. Também é possível visualizar ou criar novos POIs.

\subsection{Waze}

O Waze [11] revolucionou a criação de sistemas de recomendação de rota ao trazer o aspecto colaborativo e a cultura das redes sociais para as aplicações. Nele, o usuário possui ferramentas para editar os mapas, informando mudanças de sentido de vias, acidentes e obras públicas, bem como visualizar o posicionamento de seus amigos. Cada usuário possui um perfil único, com suas preferências e círculo de relacionamentos, e é representado por um avatar. A plataforma encoraja a participação dos membros ao premiá-los com novos avatares na medida em que interagem com o Waze e outros usuários, garantindo que o sistema de mapas esteja sempre atualizado.

Neste trabalho, destacamos como interações de mapa a capacidade de visualizar a posição atual do usuário, por meio da tecnologia de GPS, bem como a visualização de eventos de mobilidade (acidentes, interdições, má condição da via etc.) em tempo real.

\subsection{RecRoute}

O RecRoute é uma aplicação acadêmica [3], com foco na recomendação de rota para usuários do sistema público de ônibus. Além de indicar os pontos de paradas de ônibus e as principais linhas, também se preocupa com as preferências do usuário. É possível, por exemplo, procurar por veículos com acessibilidade a deficientes, trajetos mais baratos ou com poucas trocas de ônibus.

Sua interação de mapa mais inovadora consiste na criação e representação de rotas multimodais (ônibus e a pé), permitindo ao usuário utilizar mais de um tipo de meio de transporte durante o seu deslocamento. Este tipo de interação multimodal modela mais adequadamente o comportamento cotidiano de boa parte da população.

Apesar da variedade de funcionalidades e interações existentes nos aplicativos analisados, é possível notar uma lacuna quanto à exibição de todas as informações relevantes para a mobilidade, talvez motivada pela dificuldade de conciliar todos os dados nas telas dos smartphones. Os sistemas tendem a focar em meios de transporte específicos ou na exibição de apenas um conjunto das informações, o que pode não suprir perfeitamente a necessidade do usuário em seus deslocamentos diários. Desse modo, este trabalho tenta unir a exibição das funcionalidades destacadas nos aplicativos similares, dando ao usuário uma visão mais geral de suas opções de mobilidade.

\section{Princípios do Wayfinding}

A área de Wayfinding pode ser definida, segundo [7], como o estudo da facilidade com que alguém se desloca entre um ponto de interesse e outro. Isto é, refere-se a qualquer 
instrumento ou técnica que auxilie na escolha do caminho mais adequado entre duas localidades, sejam elas dois pontos distantes no planeta ou duas salas de um edifício.

O termo Wayfinding passou a ser utilizado neste contexto a partir de 1960, no livro "The Image of the City", de Kevin Lynch [8]. Desde então, passou a ser uma das áreas de interesse principalmente da Arquitetura, Design e Planejamento Urbano, através da confecção de artefatos que ajudassem na mobilidade da população dentro de um ambiente. Como exemplo destes artefatos, podemos citar desde mapas rodoviários e placas de trânsito até simbologias para pontos de interesse comuns (toaletes, restaurantes, parques etc.).

Apesar de normalmente serem aplicados na Arquitetura e Urbanismo, os conceitos do Wayfinding podem ser adaptados para sistemas de informação que utilizem dados de navegação, sobretudo àqueles voltados à mobilidade urbana, como os aplicativos de recomendação de rota.

Assim, o Wayfinding possui alguns princípios gerais [9] que podem vir a ser aplicados no contexto mobile:

- Criação de uma identidade para diferentes locais:

A rápida identificação e memorização de determinada área ajuda o usuário a se situar no espaço navegável e planejar seu deslocamento. É importante diferenciar pontos de interesse e áreas de mapas, como cidades, bairros e ruas.

- Criação de uma rota bem estruturada:

Rotas bem estruturadas devem ser contínuas, possuir início e fim claramente definidos e indicar o progresso do usuário ao longo do deslocamento.

- Criação de regiões visuais diferentes:

Delimitar algumas áreas e separá-las por características visuais diferentes, como cores, auxilia o usuário a lidar com o espaço de forma estruturada e modular. Assim, evita-se que a grande quantidade de informação presente em um mapa se torne confusa.

- Marcos para indicação de pontos de interesse e orientações:

Pontos de interesse são uma das principais formas de um ser humano se situar e memorizar um ambiente. Indicá-los claramente ajuda no entendimento do mapa e no planejamento do deslocamento. Além disso, orientações de navegação devem estar presentes, como a indicação do sentido de fluxo de vias, cruzamentos e mudanças de meio de transporte no trajeto.

- Fornecer sinais para auxílio em momentos de decisão:

É importante manter o usuário informado sobre qualquer decisão a ser tomada antes ou ao longo do trajeto, indicando também, o que o aguarda em cada alternativa.

- Utilizar visualizações de pesquisa:

Permitir ao usuário manipular diretamente o mapa e seu conteúdo é uma importante ferramenta de usabilidade. Poder observar o ambiente como um 
todo, escolher que informações estarão sendo mostradas no momento ou retirar aquelas que não serão utilizadas no trajeto torna o sistema personalizado. Assim, os dados filtrados pela preferência do usuário tornam-se de maior relevância para a navegação, acarretando numa maior compreensão do ambiente ao seu redor.

Estes princípios serão observados e respeitados na construção do protótipo proposto neste trabalho.

\section{Metodologia}

O objetivo geral desta metodologia é o desenvolvimento de um protótipo de interface de baixa fidelidade.

Para isto, foi levada em consideração a técnica cíclica de desenvolvimento de protótipos utilizada tradicionalmente pelo Design de Interação [4]. Nesta técnica, é permitido ao protótipo estar sempre em evolução, sendo validado com os usuários ao mesmo tempo em que é desenvolvido. Isso acontece principalmente pelo fato do Design de Interação ter seu foco na usabilidade e possíveis interações do protótipo. O processo completo utilizado neste trabalho pode ser observado na Figura 1 abaixo.

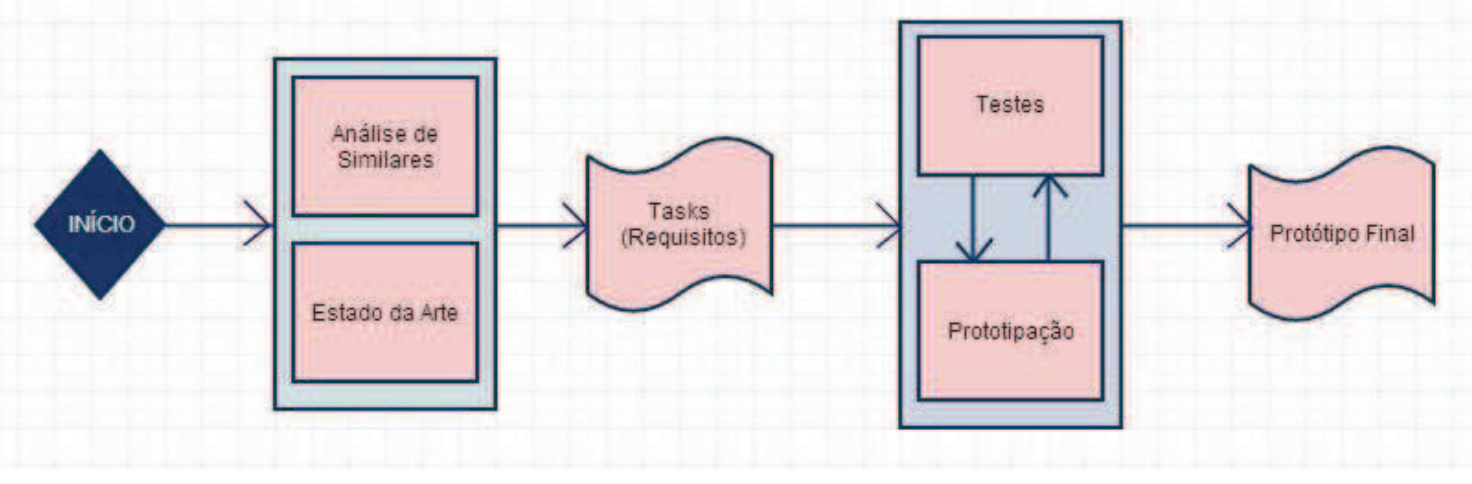

Figura 1. Fluxo de criação do protótipo.

No início do processo, é realizada uma análise de aplicativos semelhantes, bem como uma pesquisa do estado da arte na área de Wayfinding. Desse modo, são definidas as tasks a serem implementadas no protótipo. Uma task é uma tarefa de interação entre o usuário e o sistema de mapas, que já existia em algum dos aplicativos similares. Cada task será adaptada por determinado conceito de Wayfinding no protótipo, visando melhoria da usabilidade durante sua execução.

A fase de prototipação é realizada a seguir, por meio da ferramenta online Fluid UI [12]. Para garantir que as interfaces sejam visualmente agradáveis aos usuários e não atrapalhem a avaliação das adaptações de Wayfinding, a prototipação segue a definição das Oito Regras de Ouro de Shneiderman [5] e a HIG Android (Guia para Interfaces Humanas do Android) [6].

Primeiramente, o protótipo implementará as tasks em interface semelhante ao aplicativo de onde foram retiradas. Ou seja, serão mantidos fluxos de ação e elementos de tela correspondentes aos encontrados no sistema de origem da funcionalidade. Deste modo, testes iniciais poderão ser feitos a fim de criar uma base de comparação. A seguir, o protótipo passará por uma adaptação de interface, seguindo as técnicas de 
Wayfinding. Novos testes serão realizados com um grupo de voluntários, medindo a velocidade de execução de cada task e a opinião dos usuários acerca da clareza do fluxo de ação das tarefas e das informações contidas nos mapas.

As tasks implementadas foram escolhidas de maneira a representar as possíveis interações entre o usuário, o mapa e sua simbologia. Tais tasks encontram-se listadas na Figura 2, bem como o aplicativo de onde foram retiradas.

\begin{tabular}{cl}
\multicolumn{1}{c}{ Task } & \multicolumn{1}{c}{ Descrição } \\
\hline Google Maps & \\
\hline T01 & Visualização da malha de transporte \\
\hline T02 & Visualização das condiçôes de trâfego \\
\hline T03 & Visualização de POls por categoria \\
T04 & Indicação de rota por modal \\
\hline T05 & Localizaçâo e marcaçâo de pontos do mapa \\
\hline Waze & \\
\hline T06 & Visualizaçẫo da posição atual do usuário (GPS) \\
\hline T07 & Visualização de eventos de mobilidade (acidentes, buracos, via interditada...) \\
\hline RecRoute & \\
\hline T08 & Indicação de rota multimodal
\end{tabular}

Figura 2. Tasks escolhidas para implementação.

\section{Prototipação e Testes}

Nesta seção, serão apresentadas as principais funcionalidades e diferenças envolvendo as duas fases do protótipo desenvolvido, bem como a atividade de testes realizada para analisar o impacto da inserção do Wayfinding.

\subsection{Protótipo}

Algumas das primeiras adaptações encontram-se na tela inicial do sistema, como pode ser observado na Figura 3, abaixo. Em primeiro lugar, temos uma simplificação do mapa em relação ao comumente utilizado nos aplicativos de recomendação de rota. Somente as vias principais possuem indicação explícita (a não ser quando aplicado o zoom) e a indicação do sentido da rota encontra-se sempre ao lado do nome da rua a fim de diminuir a quantidade de informação exibida. Para seguir os princípios do Wayfinding, legendas foram adicionadas a todas as opções de menu, bem como uma identidade visual de cores diferentes para todas as funcionalidades.

No menu principal, no canto inferior da tela, existem as opções de criação de rota, visualização de POIs e visualização de eventos de mobilidade (como acidentes, vias interditadas e outros acontecimentos que possam afetar o deslocamento dos indivíduos no espaço). A opção de exibição da malha modal e do trânsito (que no protótipo inicial também se encontra no menu principal) foi transferida para um menu retrátil lateral, para facilitar a alternância entre as exibições. Em seu lugar, foi criada a 
opção de GPS, onde o usuário pode ativar ou desativar a exibição de sua posição atual no mapa.
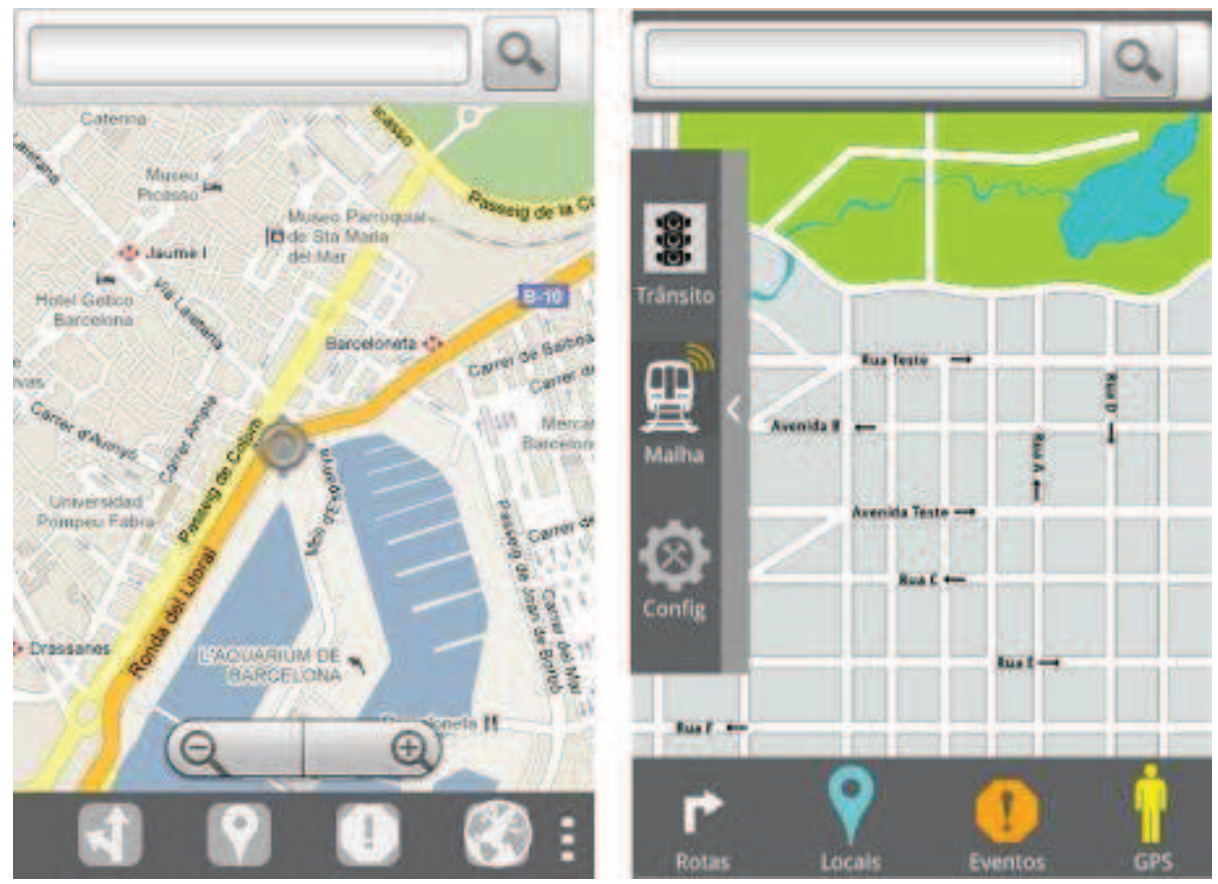

Figura 3. Tela Inicial do primeiro protótipo (à esquerda) e do adaptado (à direita).

$\mathrm{Na}$ opção de visualização de POIs, foram criados marcadores junto ao nome do estabelecimento para cada tipo de ponto de interesse (por exemplo, restaurantes são representados pelo tradicional símbolo dos talheres), facilitando a identificação por parte do usuário, como mostrado na Figura 4. Todos os pontos, ao serem clicados, exibem uma tela com informações adicionais. O mesmo princípio foi aplicado na visualização de eventos de mobilidade.
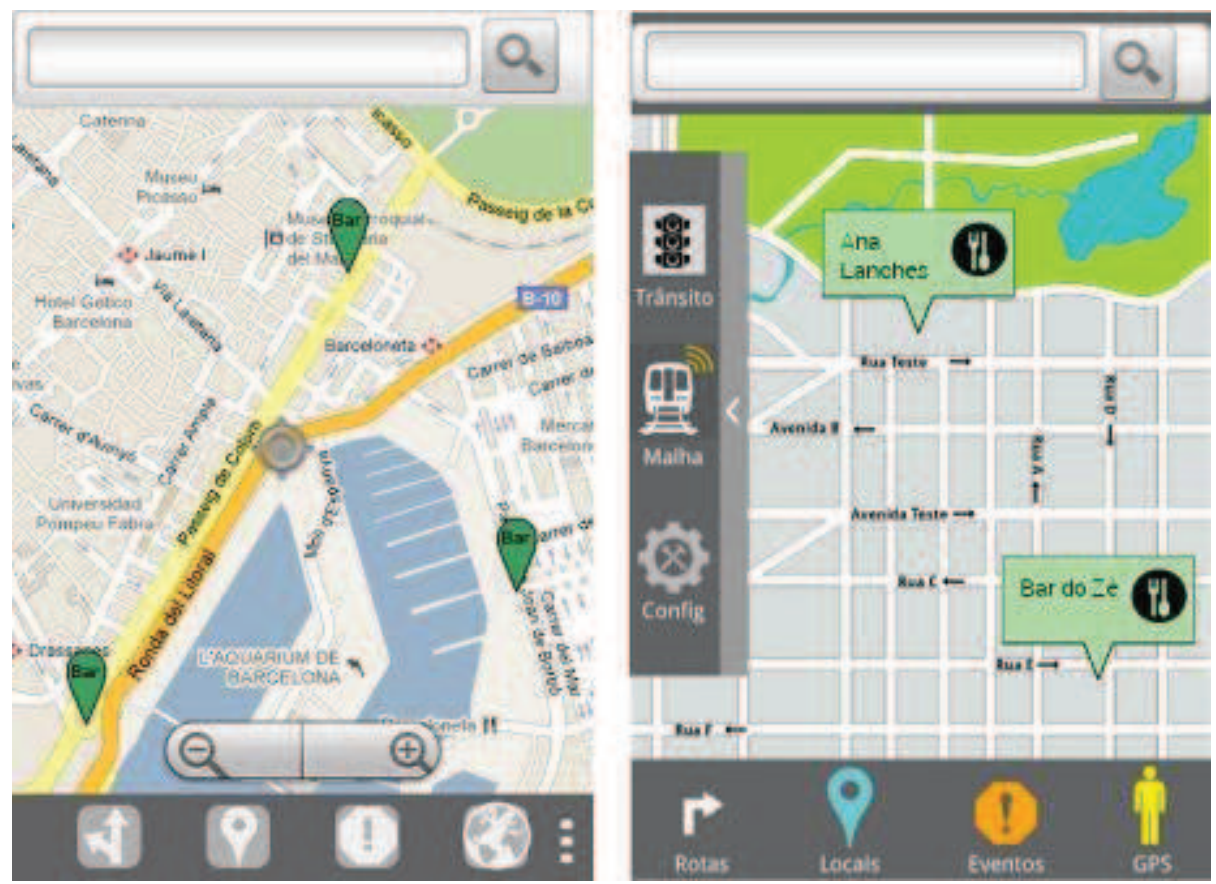

Figura 4. Indicação de POls no primeiro protótipo (à esquerda) e no adaptado (à direita). 
$\mathrm{Na}$ exibição de rotas no mapa, os pontos dinâmicos do trajeto (mudança de modal, pontos de parada na rota etc.) tornaram-se clicáveis, exibindo mais detalhes sobre o deslocamento, como por exemplo, que linhas de ônibus pegar. Assim, o usuário adquire uma abrangente visão espacial da rota, planejando melhor seu trajeto momento a momento, de maneira que o aplicativo possa guiá-lo em seu progresso entre um ponto inicial e final. Esta funcionalidade segue um dos princípios definidos anteriormente, de criação de rotas bem estruturadas e progressivas. A disposição da rota no mapa pode ser visualizada na Figura 5.
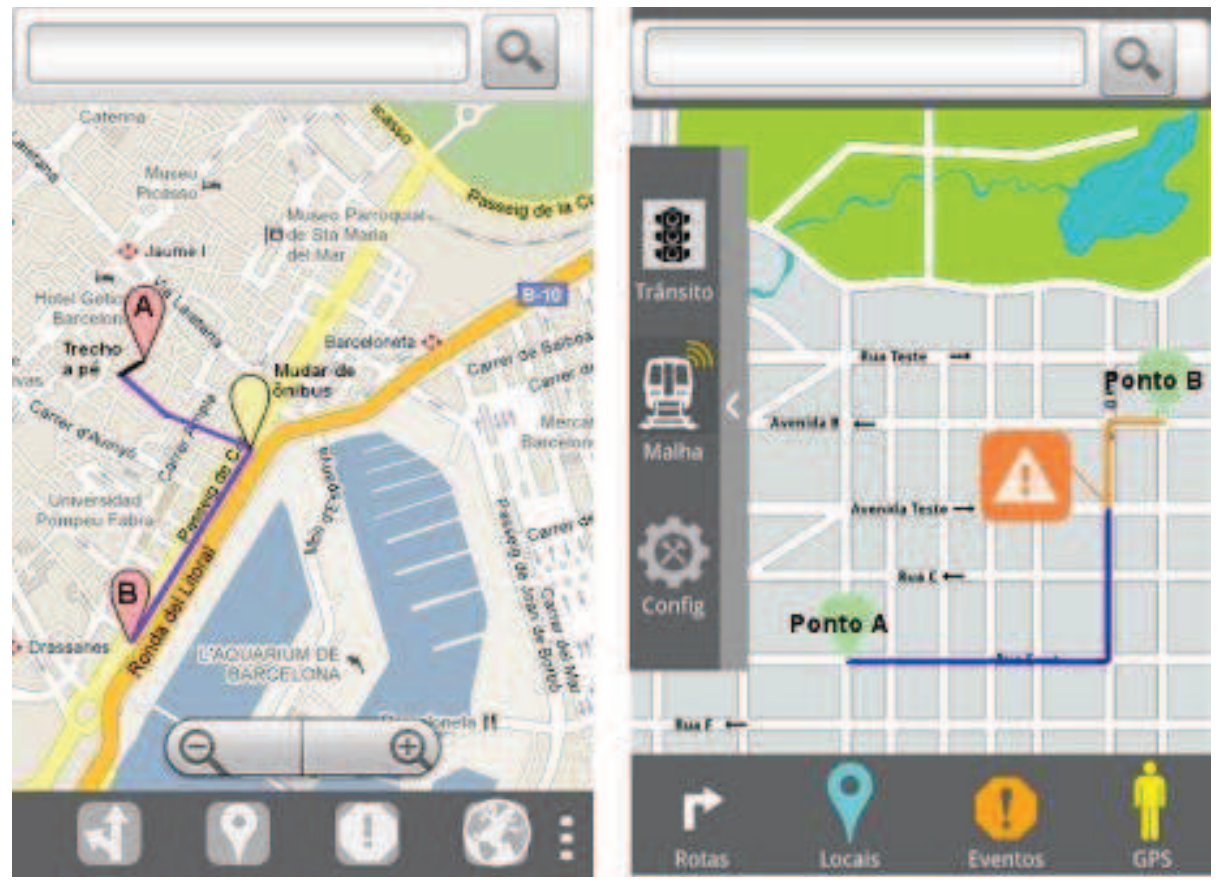

Figura 5. Exibição da rota no primeiro protótipo (à esquerda) e no adaptado (à direita).

A exibição das condições de trânsito e da malha de um modal foi adaptada de maneira a minimizar a poluição de informações presentes na tela, que acaba muitas vezes confundindo o usuário. Além de criar identidades visuais para os elementos (tipos de linha, cores e diâmetros diferentes), evitou-se ao máximo a sobreposição (linhas que atravessam uma mesma via são exibidas lado a lado, nunca sobrepostas). A capacidade de uma rápida alternância entre a visualização de trânsito, malha ou ambos permite uma visão mais clara e global do espaço. O protótipo adaptado passou também a apresentar diferenciação entre as opções de trajeto de modais públicos (como as linhas de um sistema de ônibus), tanto em cor quanto em legenda. A Figura 6 mostra a exibição destas opções nas duas fases do protótipo. 

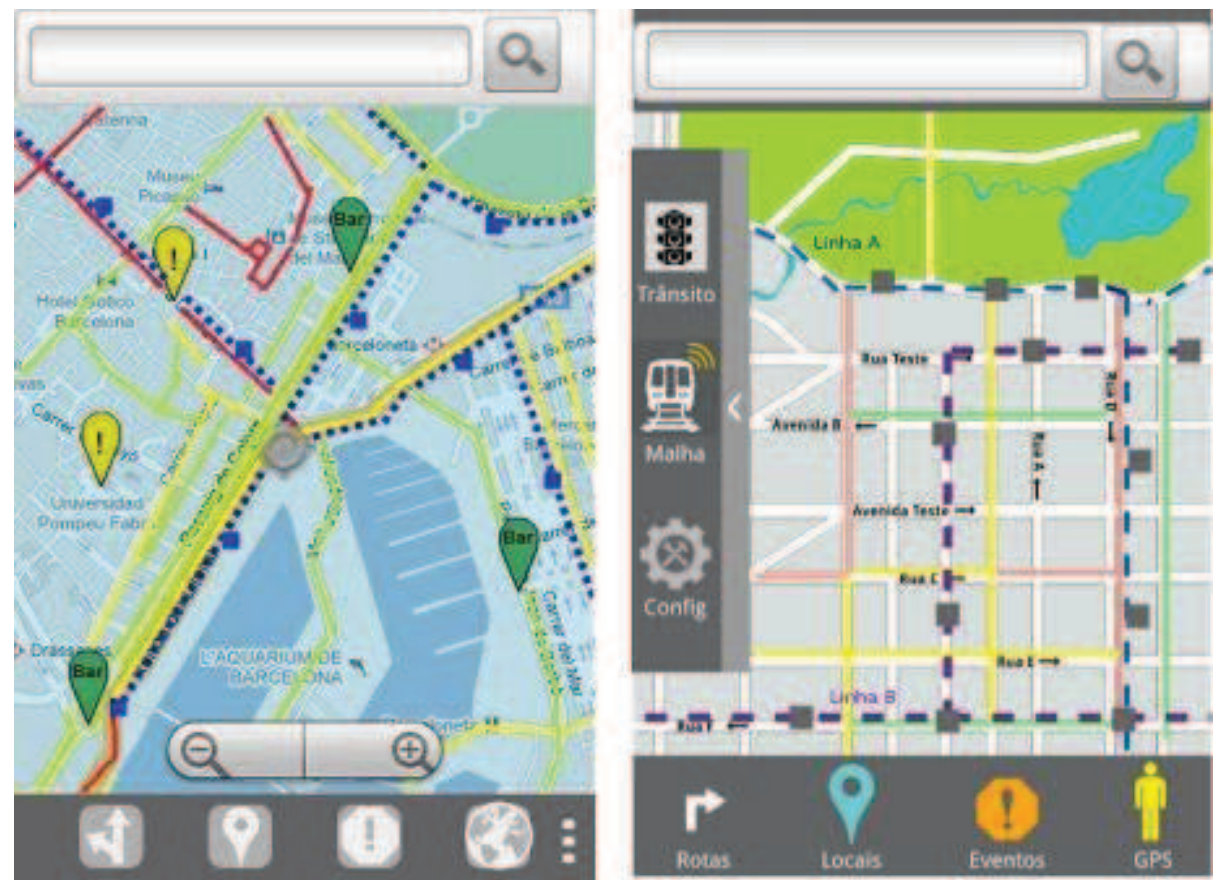

Figura 6. Malha e trânsito no primeiro protótipo (à esquerda) e no adaptado (à direita).

Além disso, na opção de configuração da malha (menu lateral), ao escolher o modal a ser exibido, a aplicação fornece as legendas que serão utilizadas em sua representação (Figura 7). Esta funcionalidade foi adicionada em conformidade com as noções de Wayfinding de criar marcos eficazes de orientação para o usuário, e não estava presente na primeira versão do protótipo.

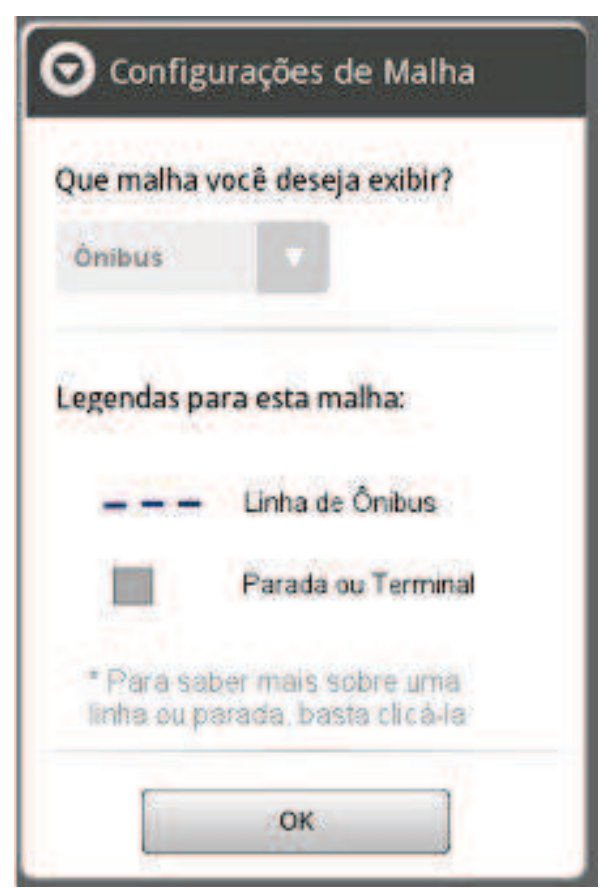

Figura 7. Tela de Configuração exibe legendas da malha no protótipo adaptado.

Algumas outras funções menores também foram adicionadas ao protótipo, como a opção de exibir o endereço da posição atual do GPS e pré-visualizar as opções de rota possíveis entre dois pontos no mapa. 


\subsection{Testes}

O teste proposto para este projeto consiste na realização de quatro tarefas, escolhidas de maneira a prover a interação do usuário com cada uma das tasks implementadas pelo protótipo. As tarefas consistem em:

- Tarefa 1: Exibir no mapa todos os POIs da categoria "Restaurante" e encontrar uma rota até um dos estabelecimentos.

- Tarefa 2: Exibir no mapa todos os Eventos de Mobilidade da categoria "Acidentes" e exibir os detalhes sobre um dos eventos.

- Tarefa 3: Exibir simultaneamente no mapa as informações sobre o tráfego e a malha para o modal "Ônibus".

- Tarefa 4: Escolher um ponto específico no mapa e criar uma rota até a localização deste ponto utilizando sua posição atual de GPS.

Os testes foram realizados com 10 voluntários, onde 5 pessoas realizaram o experimento com o protótipo inicial e as outras 5 foram submetidas a teste com o protótipo adaptado. O perfil dos voluntários foi composto em $40 \%$ por mulheres e $60 \%$ homens, com idades entre 21 e 55 anos, onde $50 \%$ realiza seus deslocamentos por meio de transporte público e $50 \%$ faz uso de veículos particulares. Foram medidos o tempo de execução das tarefas e a avaliação (com critério qualitativo de 1 a 5) de cada usuário sobre a qualidade da informação visual apresentada na tela e clareza do fluxo de ação de cada tarefa. Também foram colhidas opiniões e críticas ao protótipo por meio de uma entrevista conduzida após a realização do experimento, para identificação de possíveis melhorias.

A Figura 8 apresenta um gráfico comparativo do tempo de execução médio de cada tarefa entre os dois grupos de usuários teste. A seguir, na Figura 9, outro gráfíco mostra a nota média da avaliação dada pelos usuários para cada tarefa, tanto para o protótipo inicial quanto para o adaptado.

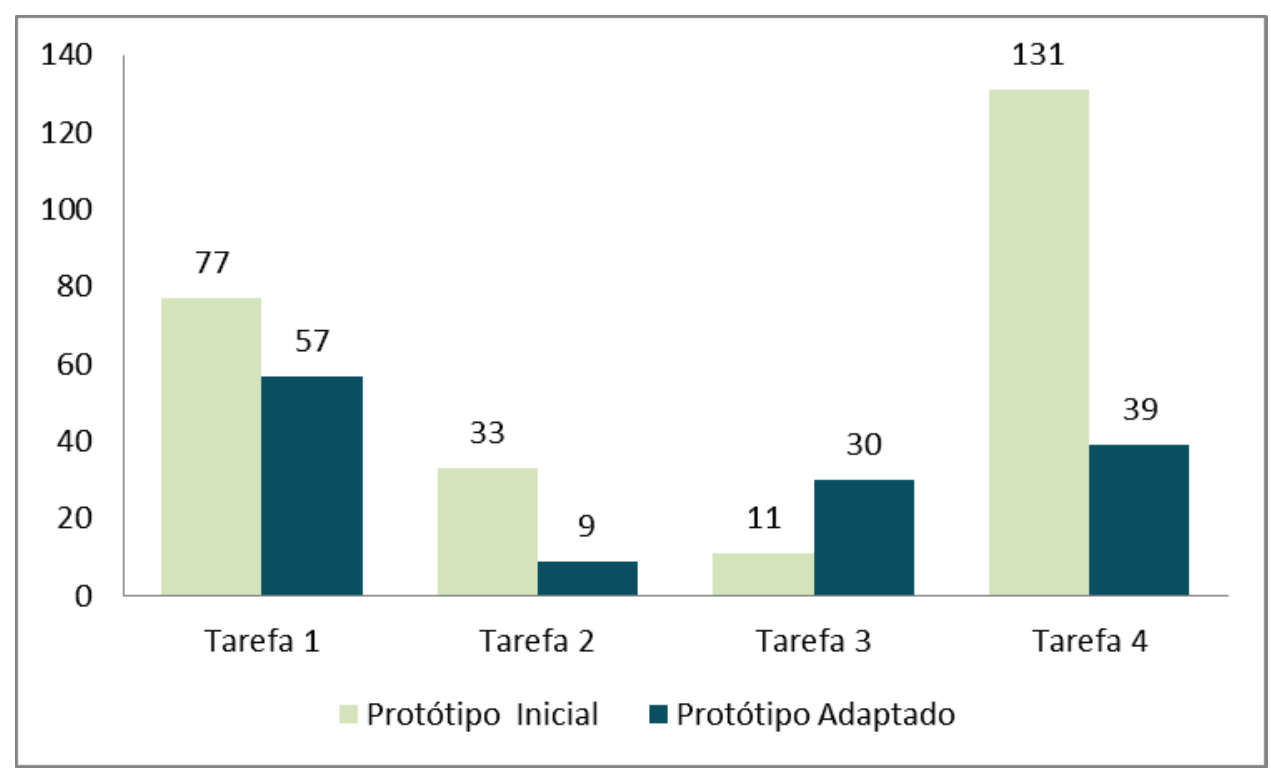

Figura 8. Tempo de execução médio para cada tarefa do experimento (em segundos). 


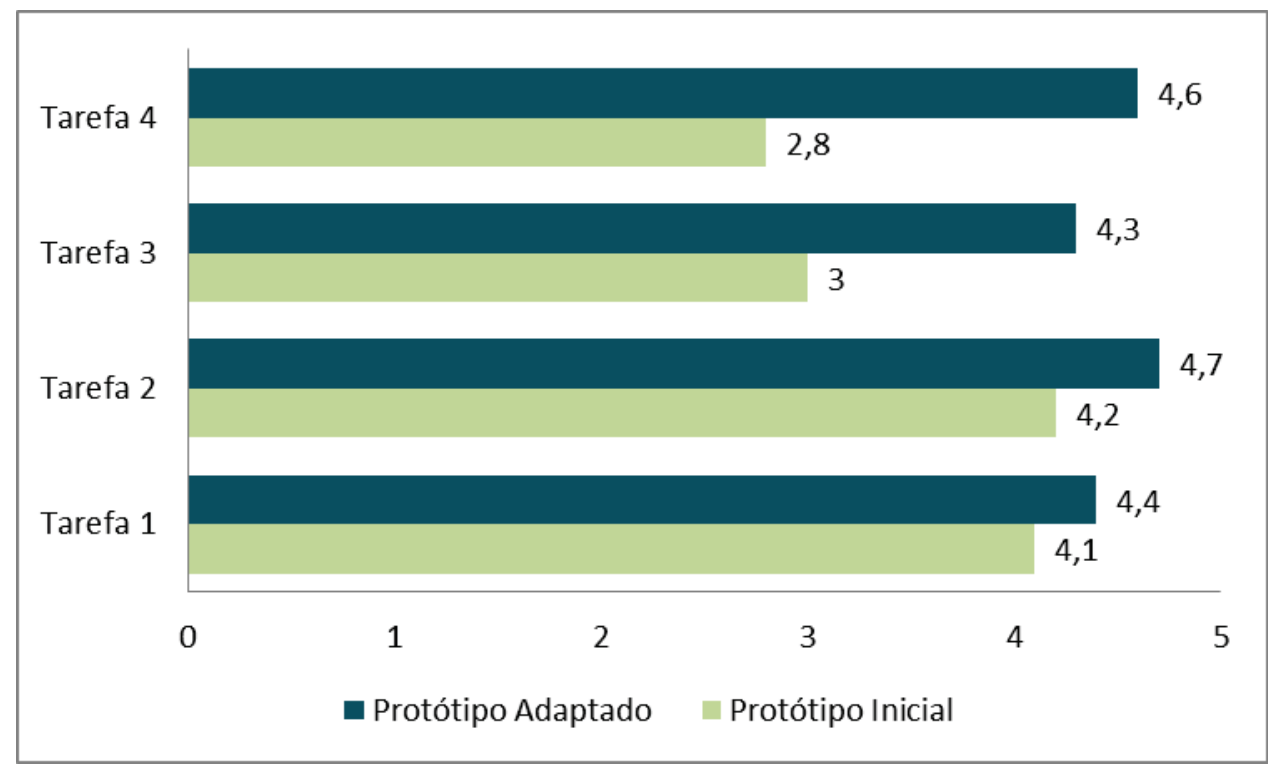

Figura 9. Nota de avaliação média para cada tarefa do experimento.

É possível notar uma melhora substancial no tempo médio de execução das tarefas, com exceção da Tarefa 3, onde o tempo médio sofre um aumento. A avaliação média de todas as tarefas realizadas com o protótipo adaptado também apresenta melhoria em relação ao protótipo inicial.

\section{Conclusões e Trabalhos Futuros}

A tecnologia mobile tornou-se uma grande aliada nas ferramentas de mobilidade urbana, sobretudo nos aplicativos de recomendação de rota. No entanto, a quantidade e complexidade de informações que precisam ser apresentadas ao usuário em espaços reduzidos de tela pode representar um empecilho à usabilidade de sistemas do gênero.

Este trabalho teve por objetivo a criação de um protótipo de baixa fidelidade para a investigação de possíveis impactos na usabilidade de aplicativos mobile de recomendação de rota após a aplicação do Wayfinding na representação da interface. É possível notar, através dos resultados dos experimentos, que a adaptação do protótipo permitiu uma melhora do tempo de execução na maior parte das interações, bem como uma maior satisfação dos usuários com a forma de apresentação das funcionalidades propostas.

Como trabalho futuro, podemos destacar a melhora das funcionalidades do protótipo levando em consideração as sugestões recolhidas durante os testes com usuários, de maneira a tentar diminuir o tempo de execução e aumentar a avaliação média das tarefas executadas. Também é desejável a implementação de um aplicativo real, para testes mais elaborados acerca da usabilidade da interface adaptada por Wayfinding.

\section{Referências}

[1] NOKIA CORPORATION. Mobile Game Graphics: Overcoming the Small Screen Challenge. 2007. Disponível em:

$<$ http://developer.nokia.com/info/sw.nokia.com/id/efbc3add-2217-4e51-bdcb- 
ddb7961cd9ad/Mobile_Game_Graphics_Overcoming_the_Small_Screen_Challenge _v1_0_en.pdf.html>. Acesso em: 30 dez. 2013.

[2] CENTER FOR INCLUSIVE DESIGN AND ENVIRONMENTAL ACCESS. University At Buffalo, The State University Of New York. Universal Design New York: Wayfinding. 2001. Disponível em: <http://idea.ap.buffalo.edu/udny/Section41c.htm>. Acesso em: 30 dez. 2013.

[3] TITO, A. O.; RISTAR, A.; SANTOS, L.; FILHO, L. A.; TEDESCO, P.; SALGADO, A. C. RecRoute: Uma Proposta de Aplicativo para Recomendação de Rotas de Ônibus Utilizando Informações Contextuais dos Usuários. 2013. Disponível em: $<$ http://www.cin.ufpe.br/ ubibus/artigos/proposta_recRoute.pdf $>$. Acesso em: 20 nov. 2013.

[4] PREECE, J.; SHARP, H.; ROGERS, Y. Interaction design: Beyond humancomputer interaction. EUA: John Wiley \& Sons, Inc, 2002.

[5] SHNEIDERMAN, B. Designing the user interface: Strategies for effective humancomputer interaction. Michigan: Pearson Education, 2003.

[6] ANDROID OPEN SOURCE PROJECT. Design: Android Developers. Disponível em: $<$ http://developer.android.com/design/index.html $>$. Acesso em: 01 jan. 2014.

[7] QUEENSLAND GOVERNMENT. Building Guidelines for Queensland Mental Health Facilities. 1996. Disponível em:

<http://www.health.qld.gov.au/cwamb/mhguide/>. Acesso em: 05 jan. 2014.

[8] LYNCH, Kevin. The Image of the City. EUA: Mit Press, 1960.

[9] FOLTZ, Mark A. Designing Navigable Information Spaces. 1998. Massachusetts Institute of Technology. Disponível em:

$<$ http://www.ai.mit.edu/projects/infoarch/publications/mfoltz-thesis/>. Acesso em: 05 jan. 2014.

[10] GOOGLE. Google Maps. Disponível em: < https://maps.google.com.br/>. Acesso em: 02 fev. 2014.

[11] WAZE MOBILE. Waze. Disponível em: <https://www.waze.com/pt-BR>. Acesso em: 02 fev. 2014.

[12] FLUID SOFTWARE. Fluid UI. Disponível em: $<$ https://www.fluidui.com/>. Acesso em: 02 fev. 2014. 